\title{
Performance Analysis of Hybrid Phase Shift Keying (HPSK) over Generalized Nakagami Fading Channels
}

\author{
Mahmoud Youssuf* and M.Zaki Abdelmageed $^{* *}$
}

\begin{abstract}
Bit Error Rate (BER) of Hybrid Phase Shift Keying (HPSK) modulation systems is shown to outperform OQPSK and GMSK by $3 \mathrm{~dB}$. HPSK performance through Nakagami Fading Channels is also considered.
\end{abstract}

Keywords: Hybrid Phase Shift Keying (HPSK), and Bit Error Rate (BER)

\section{Introduction}

Hybrid Phase Shift Keying (HPSK) is used in WCDMA systems thanks to its low peak-toaverage power ratio. This low ratio of peak-to-average power results in reducing the number of zero crossing of phase transitions of the output transmitted signal. In this paper we prove that HPSK outperforms other Quadrature modulation techniques suck as OQPSK by $3 \mathrm{~dB}$.

The paper is organized as follows: Section II describes the HPSK constellation in case of two channels at different amplitudes. Section III derives an expression for the conditional probability of error of HPSK modulated signal over AWGN. In section IV we apply the obtained expression in evaluating the performance of HPSK modulated signal over a generalized Nakagmi $-\mathrm{m}$ channel. Finally section V includes numerical results and comments.

\section{The HPSK Constellation}

The Hybrid Phase Shift Keying (HPSK) has been proposed as the spreading technique for WCDMA to eliminate the zero crossings for every other signal transition and to eliminate the $0^{\circ}$-degree phase shift transitions for every other chip point, as well as to improve the BER measure of the DS-WCDMA system performance.

In $3 \mathrm{G}$ systems the mobile Station (MS) can transmit more than one channel. The different channels are assigned to either I or Q path.

In the case of transmitting only two channels as in figure (1) one of the channels (DPDCH or RPilot) is applied to the I path and the other channel (DPCCH or R-FCH) is applied to the Q path [1].

\footnotetext{
* Egyptian Armed Forces

** Faculty of Engineering, Azhar University, Cairo, Egypt 
Additional high data rate channels are combined alternatively on the I or Q paths. Each channel is spread by a different orthogonal even-numbered Walsh code.In the general case the channels can be at different power levels as in figure (2) which maps onto a rectangular four Quadrature amplitude Modulation (QAM) constellation.

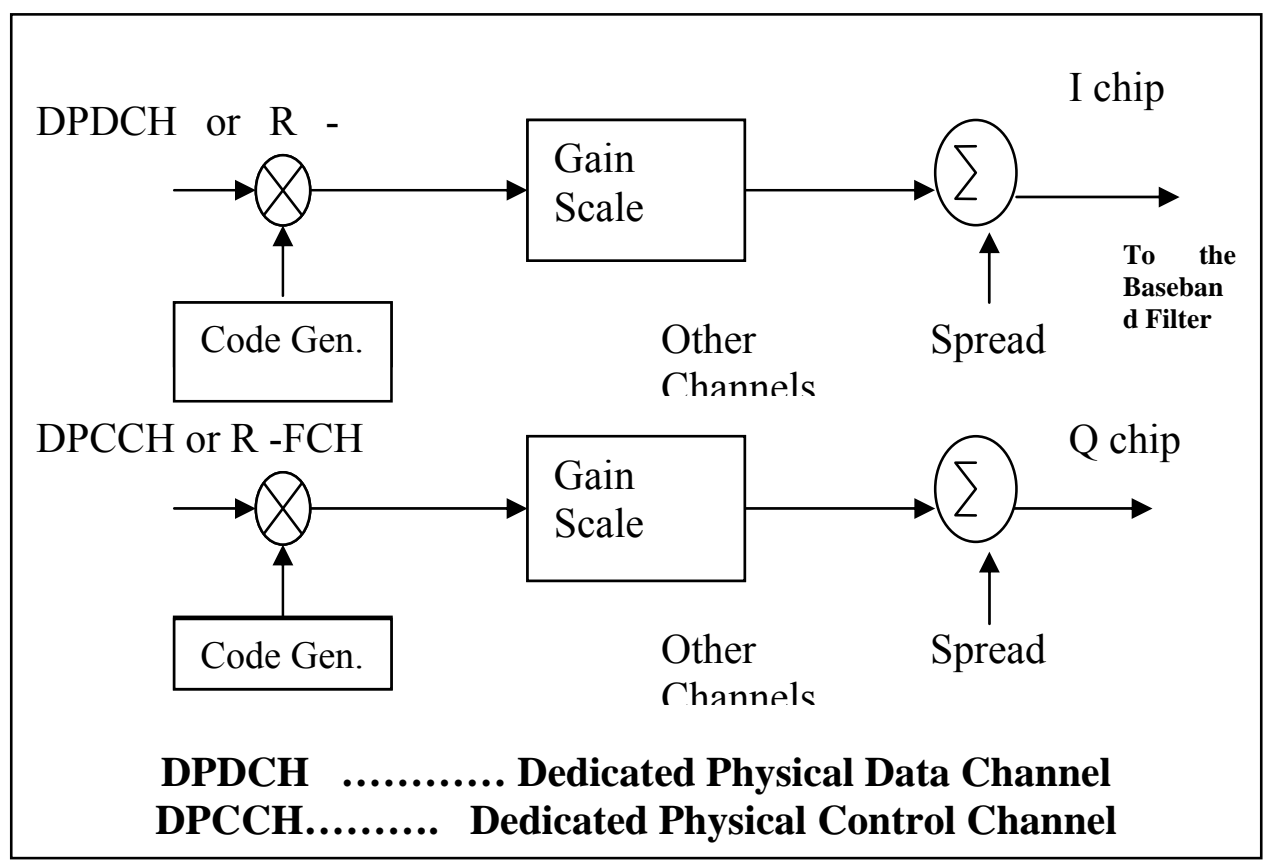

Fig.1 The basic reverse channel structure of 3G system

The QAM signal waveforms may be expressed as:

$$
\begin{aligned}
& \mathrm{S}_{\mathrm{m}}(\mathrm{t})=\operatorname{Re}\left\{\mathrm{A} \mathrm{e}{ }^{\mathrm{j} \theta_{\mathrm{m}}} \mathrm{g}(\mathrm{t}) \mathrm{e}^{\mathrm{j} 2 \Pi \mathrm{f}_{\mathrm{c}} \mathrm{t}}\right\} \ldots \ldots \ldots \ldots \ldots \ldots \ldots \ldots \ldots \ldots \ldots \ldots \ldots \ldots \ldots \ldots \ldots \ldots \ldots \ldots \ldots \\
& =A_{c h} g(t) \cos (2 \pi f c t+\theta m) \\
& =A_{c h} g(t)[\cos (2 \pi f c t) \cos (\theta m)-\sin (2 \pi f c t) \sin (\theta m)]
\end{aligned}
$$

where $\mathrm{A}_{\mathrm{ch}}=\sqrt{I_{c h}^{2}+Q_{c h}^{2}} \quad, \mathrm{I}_{\mathrm{ch}}$ and $\mathrm{Q}_{\mathrm{ch}}$ are the information bearing signals amplitudes of I path channel and $Q$ path channel respectively.

$\mathrm{g}(\mathrm{t}) \ldots$. is the pulse shape signal

$$
\theta_{\mathrm{m}}=\tan ^{-1} \frac{\mathrm{Q}_{\mathrm{ch}}}{\mathrm{I}_{\mathrm{ch}}}
$$

So the QAM signal waveform may be viewed as a linear combination of two orthogonal wave forms f1(t) and $\mathrm{f}(\mathrm{t})$ such that [2] :

$$
S_{m}(t)=S_{m 1} f_{1}(t)+S_{m 2} f_{2}(t) .
$$


Where, $\mathrm{S}_{\mathrm{m} 1}$ and $\mathrm{S}_{\mathrm{m} 2}$ are the component of the two dimensional vector $\mathrm{S}_{\mathrm{m}}: \quad \mathrm{S}_{\mathrm{m}}=\left[\begin{array}{ll}\mathrm{S}_{\mathrm{m} 1} & \mathrm{~S}_{\mathrm{m} 2}\end{array}\right]$,

$$
\begin{aligned}
& \mathrm{S}_{\mathrm{m}}=\left[\begin{array}{ll}
\mathrm{I}_{\mathrm{ch}} & \mathrm{Q}_{\mathrm{ch}}
\end{array}\right] \\
& A_{c h}=\sqrt{I_{c h}^{2}+Q_{c h}^{2}} \quad, \quad \theta_{m}=\tan ^{-1} \frac{Q_{c h}}{I_{c h}} \ldots
\end{aligned}
$$

where: $\quad \mathrm{A}_{\mathrm{ch}} \ldots . .$. is the amplitude of the chip signal $\theta_{m} \ldots$. is the phase of the signal vector and it varies with $\mathrm{m} \quad \mathrm{m}=1,2,3,4$

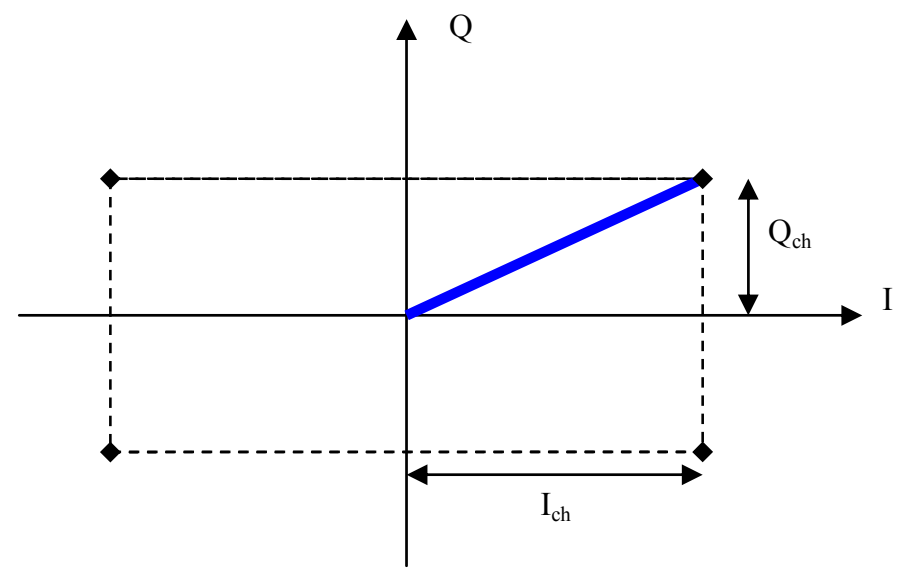

Fig.2 4-QAM constellation for two channels at different amplitudes

in the 4-QAM according to the position of the vector point and according to the value of Ich and Qch

$$
\therefore \mathrm{S}_{\mathrm{m}}=\left[\begin{array}{ll}
\mathrm{A}_{\mathrm{ch}} \cos \theta_{\mathrm{m}} & \mathrm{A}_{\mathrm{ch}} \sin \theta_{\mathrm{m}}
\end{array}\right]
$$

In the reverse link of DS-CDMA systems the Ich and Qch are complex scrambled with a complex scrambling signal $(\mathrm{Is}+\mathrm{jQs})$ as in figure 3 .

The final I and Q signals are produced mathematically by the multiplication of the two complex signals; the complex data signal $\left(\mathrm{I}_{\mathrm{D}}+\mathrm{j} \mathrm{Q}_{\mathrm{D}}\right)$ which has already spread into chips $\left(\mathrm{I}_{\mathrm{ch}}+\mathrm{j} \mathrm{Q}_{\mathrm{ch}}\right)$, and the complex scrambling signal $\left(\mathrm{I}_{\mathrm{s}}+\mathrm{j} \mathrm{Q}_{\mathrm{s}}\right)$ so the final I and $\mathrm{Q}$ signals are:

$$
\begin{aligned}
& I=I_{c h} \cdot I_{s}-Q_{c h} \cdot Q_{s} \\
& Q=I_{c h} \cdot Q_{s}-Q_{c h} I_{s} \\
& I+J Q=\left(I_{c h} I_{s}-Q_{c h} Q_{s}\right)+j\left(I_{c h} Q_{s}+Q_{c h} I_{s}\right)=A_{c h} A_{s} e^{j(\phi c h+\phi s)}
\end{aligned}
$$




$$
\begin{aligned}
& I=A_{c h} \cos \theta_{m} \cdot A_{s} \cos \frac{\pi}{M}(2 n-1)-A_{c h} \sin \theta_{m} \cdot A_{s} \sin \frac{\pi}{M}(2 n-1) \\
& I=A_{c h} A_{s} \cos \left(\frac{\pi}{M}(2 n-1)+\theta_{m}\right)=A \cos \left(\frac{\pi}{M}(2 n-1)+\theta_{m}\right) \ldots \ldots \ldots \ldots \ldots . . . \\
& Q=A_{c h} \cos \theta_{m} \cdot A_{s} \sin \frac{\pi}{M}(2 n-1)-A_{c h} \sin \theta_{m} \cdot A_{s} \cos \frac{\pi}{M}(2 n-1) \\
& Q=A_{c h} A_{s} \sin \left(\frac{\pi}{M}(2 n-1)+\theta_{m}\right)=A \sin \left(\frac{\pi}{M}(2 n-1)+\theta_{m}\right) \ldots \ldots \ldots \ldots \ldots \ldots
\end{aligned}
$$

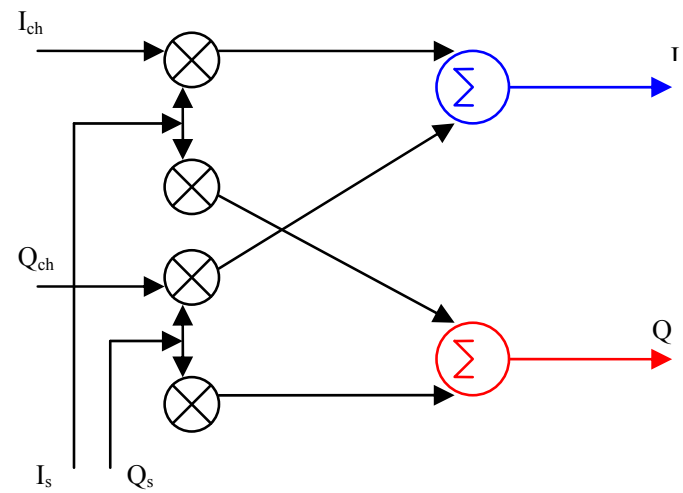

Fig.3 Complex Scrambling Of HPSK

Since final constellation is formed by complex multiplication of the two signals of chip constellation and scrambling constellation which is always QPSK constellation as in Figure (4)-, then:

from (9) and (10) we conclude that the final signal $(\mathrm{I}+\mathrm{jQ})$ is a QPSK constellation with two dimensional vector representation $\mathrm{S}_{\mathrm{mn}}$ where :

$S_{m n}=\left[A \cos \left(\frac{\pi}{M}(2 n-1)+\theta m\right) \ldots . . A \sin \left(\frac{\pi}{M}(2 n-1)+\theta m\right)\right] \ldots$

Where $\mathrm{A}=$ Ach.As $; \mathrm{n}=1,2,3,4 ; \mathrm{m}=1,2,3,4$ and $\mathrm{M}=4$

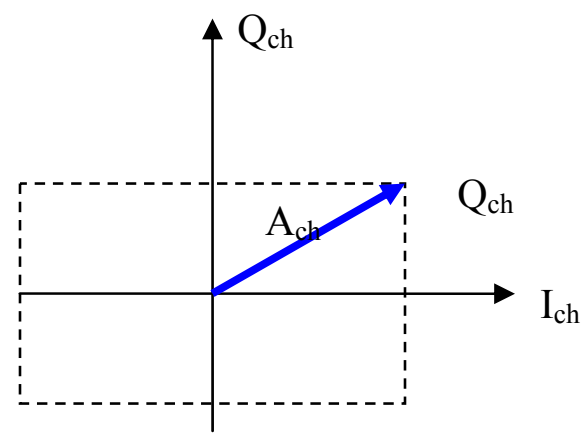

Chip constellation

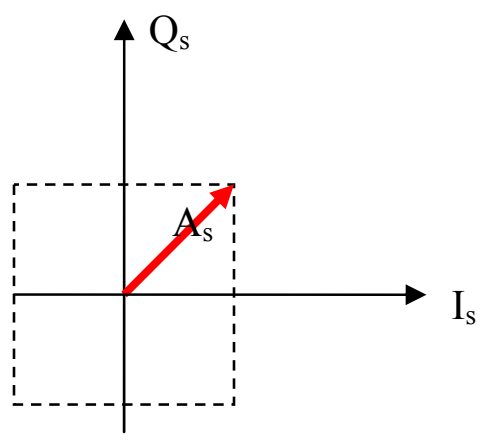

Scrambling constellation

Fig.4 the chip constellation and the scrambling constellation 
This new constellation has points that rotate according to the angle: $\frac{\pi}{4}(2 n-1)+\theta_{m}$, while $\theta_{m}$ changes according to the value of Ich and Qch for example if $n=1$, the point (Ich , Qch) will be transferred by the angle equal to $\left(45 \mathrm{o}+\theta_{m}\right)$. So the new constellation is really an eight point constellation with two independent QPSK constellation as shown in Figure (5) according to the value of $\theta_{m}$.

One of these two constellations corresponds to $\theta_{m}>45^{\circ}$ and it rotates by angle equal to $\phi_{1}=\left(\theta m-45^{\circ}\right)$ from the original axes.

The other QPSK constellation corresponds to $\theta_{m}<45^{\circ}$ and rotates with angle equal to $\phi_{2}=-\left(\theta_{m}-45^{\circ}\right)$ from the original axes.

So the new final constellation consists of two independent QPSK constellations with complex scrambling.

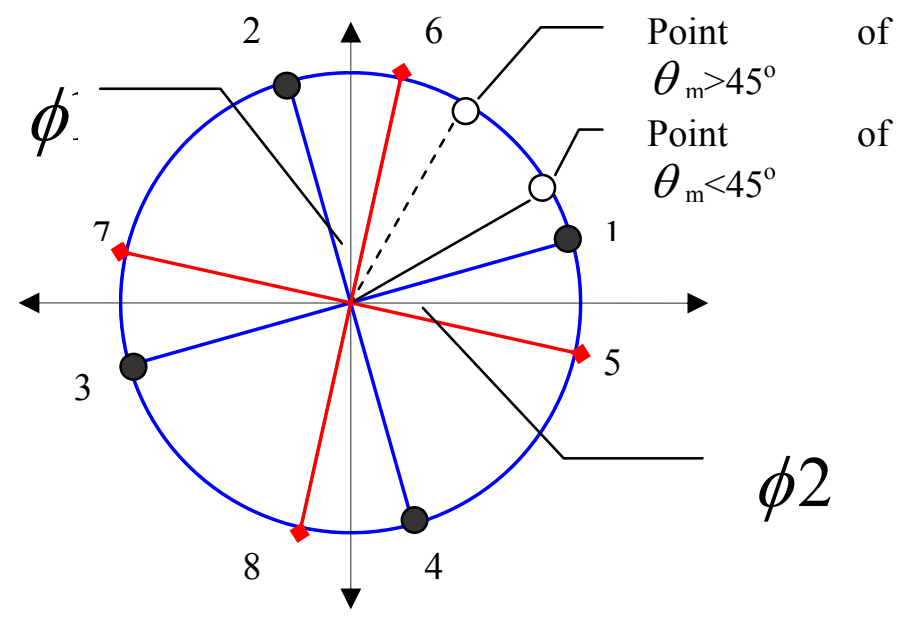

Fig.5 the final constellation of the scrambled chip of different channel amplitudes

The vector representation of the first constellation is:

$S_{n}=\left[A \cos \left[\frac{2 \pi}{M}(n-1)+\phi_{1}\right] \quad A \sin \left[\frac{2 \pi}{M}(n-1)+\phi_{1}\right]\right]$

And the vector representation of the second constellation is:

$$
S_{n}{ }^{\prime}=\left[A \cos \left[\frac{2 \pi}{M}(n-1)-\phi_{2}\right] \quad A \sin \left[\frac{2 \pi}{M}(n-1)-\phi_{2}\right]\right]
$$

So the constellation of the two channels generally has eight points distributed around a circle with the angular distribution determined by the relative levels of the two channels signals 


\section{Probability of error of HPSK modulated signal over AWGN}

We concluded in the previous section that the final constellation of the two channels at different amplitudes consists of two independent QPSK constellations with complex scrambling. The average value of the amplitude of the new constellation is $\sqrt{2}$ times the value of the amplitude of the traditional QPSK. To obtain the BER of the HPSK modulated signal as a measure of its performance we will assume that this signal is transmitted over an additive white Gaussian noise (AWGN) channel. The received signal is demodulated with correlated demodulator or a matched filter demodulator and produces the vector

$\mathrm{r}=[\mathrm{r} 1, \mathrm{r} 2, \mathrm{r} 3 \ldots \mathrm{rN}]$

where the demodulator decomposes the received signal and the noise into $\mathrm{n}$ - dimensional vector. The vector $r$ contains all the relevant information in the received signal waveform to obtain the optimum decision rule based on the observations vector $r$. The optimum detector is designed to make a decision on the transmitted signal in each signal interval based on the observation of the vector $r$ in each interval such that the probability of correct decision is maximized. The decision criterion is based on selecting the signal corresponding to the maximum of the set of posterior probabilities $\{\mathrm{P}(\mathrm{Sm} / \mathrm{r})\}$ whish may be expressed as:

$$
P\left(S_{m} / r\right)=\frac{P\left(r / S_{m}\right) \cdot P\left(S_{m}\right)}{P(r)}
$$

where: $\mathrm{P}(\mathrm{r} / \mathrm{Sm})$ is the conditional probability pdf of the observed vector given $\mathrm{Sm}$ and $\mathrm{P}(\mathrm{Sm})$ is the prior probability of the $\mathrm{mth}$ signal being transmitted $(\mathrm{m}=1,2,3,4)$.

$\mathrm{P}(\mathrm{r})$ can be expressed as:

$$
P(r)=\sum_{m=1}^{M} P\left(r / S_{m}\right) \cdot P\left(S_{m}\right)
$$

And since the denominator in (14) is independent of which signal is transmitted then the decision rule based on finding the signal that maximizes $\mathrm{P}(\mathrm{Sm} / \mathrm{r})$ is equivalent to finding the signal that maximizes $\mathrm{P}(\mathrm{r} / \mathrm{Sm})$ knowing that the $\mathrm{M}$ signals are equally probable for all $\mathrm{M}(\mathrm{P}(\mathrm{Sm})=1 / \mathrm{M})$.

The decision based on the maximum of the conditional $\mathrm{Pdf}(\mathrm{P}(\mathrm{r} / \mathrm{Sm}))$ or any monotonic function of it is called maximum likelihood (ML) criterion. So the likelihood function in the AWGN channel is given generally by:

$$
P\left(r / S_{m}\right)=\left(\frac{1}{\pi N_{0}}\right)^{N / 2} \exp \left[-\sum_{k=1}^{N} \frac{(r k-S m k)^{2}}{N_{0}}\right]
$$

where

N0.... Is the noise power spectral density.

$\mathrm{N}$......the dimesion of the received vector $\mathrm{r}$

rk ..... the Kth components of the received vector $\mathrm{r}$

Smk ...The kth component of the mth transmitted signal

Taking the natural logarithm of equation (16):

$\ln P\left(r / S_{m}\right)=-\frac{1}{2} N \ln \left(\pi N_{0}\right)-\frac{1}{N_{0}} \sum_{k=1}^{N}\left(r_{k}-S_{m k}\right)^{2}$

The maximum of $\ln \mathrm{P}(\mathrm{r} / \mathrm{Sm})$ over $\mathrm{Sm}$ is equivalent to finding the signal $\mathrm{Sm}$ that minimizes the Euclidean distance $\mathrm{D}(\mathrm{r}, \mathrm{Sm})$ where : 


$$
D\left(r, S_{m}\right)=\sum_{k=1}^{N}\left(r_{k}-S_{m k}\right)^{2}
$$

And it is called the distance metrics

By expanding (18) and ignoring the term $|r|^{2}\left(|r|^{2}=\sum_{k=1}^{N} r_{k}{ }^{2}\right)$ which is common for all decisions and ignoring the term $\left|S_{m}\right|^{2}\left(\left|S_{m}\right|^{2}=\sum_{k=1}^{N} S_{m k}{ }^{2}\right)$ because all the signals have the same energy the metric $\mathrm{D}(\mathrm{r}, \mathrm{Sm})$ reduces to:

$$
D^{\prime}\left(r, S_{m}\right)=-2 \sum_{k=1}^{N}\left(r_{k}-S_{m k}\right)=-2 r S_{m}
$$

Minimizing the metric $D^{\prime}\left(r, S_{m}\right)$ is equivalent to maximizing the metric

$$
\begin{aligned}
\mathrm{C}\left(\mathrm{r}, \mathrm{S}_{\mathrm{m}}\right)=- & D^{\prime}\left(r, S_{m}\right) \\
\mathrm{C}\left(\mathrm{r}, \mathrm{S}_{\mathrm{m}}\right) & =-2 r . S_{m}
\end{aligned}
$$

The optimum maximum likelihood (ML) detector computes a set of $\mathrm{M}$ correlation metrics $\mathrm{C}(\mathrm{r}, \mathrm{Sm})$ and selects the signal corresponding to the largest correlation metric [3] .

Applying this metric in our study case, $r$ is the received signal vector $r=\left[\begin{array}{lll}r 1 & r 2\end{array}\right]$ which is projected onto each of the four possible transmitted signals vectors $\mathrm{Sm}$ for $\mathrm{m}=1,2,3,4$ where $\mathrm{M}=4$

We can consider that the correlation detector in the case of HPSK modulated signal is equivalent to a phase detector that computes the phase of the received signal from ' $r$ ' and selects the signal Sm whose phase is closest to ' $r$ '.

Since the phase of $r$ is:

$$
\theta_{r}=\tan ^{-1} \frac{r_{2}}{r_{1}}
$$

The probability of error can be computed if we determine the Power density function of $\theta_{r}$. We consider the case in which the transmitted signal phase is equal to zero as in Figure (6) which corresponds to the transmitted vector

$S_{1}=\left[\begin{array}{ll}\sqrt{\varepsilon_{s}} & 0\end{array}\right]$

Where $\sqrt{\varepsilon_{s}}$ is the energy of the transmitted HPSK signal S1(t)

The received signal vector has two components $\mathrm{r} 1$ and $\mathrm{r} 2$

$$
\mathrm{r}=\left[\begin{array}{ll}
\mathrm{r} 1 & \mathrm{r} 2
\end{array}\right] \quad \mathrm{r}=\left[\begin{array}{ll}
\sqrt{\varepsilon_{s}}+n_{1} & \mathrm{n} 2
\end{array}\right]
$$

Where $\mathrm{n} 1$ and $\mathrm{n} 2$ are jointly Gaussian random variables with mean and variances $\mathrm{E}(\mathrm{r} 1)=\sqrt{\varepsilon_{s}}, \mathrm{E}(\mathrm{r} 2)=0$ and $\quad \sigma \mathrm{r} 12=\sigma \mathrm{r} 22=\sigma \mathrm{r} 2=1 / 2 \mathrm{~N} 0$. Consequently, The joint Pdf of $\mathrm{r} 1$ and $\mathrm{r} 2$ is: 


$$
\begin{aligned}
& P\left(r_{1}, r_{2}\right)=\left(\frac{1}{\pi N_{0}}\right)^{1} \exp \left[-\sum_{k=1}^{2} \frac{\left(r_{k}-S_{m k}\right)^{2}}{N_{0}}\right] . \\
& P\left(r_{1}, r_{2}\right)=\left(\frac{1}{\pi N_{0}}\right) \exp \left[-\frac{\left(r_{1}-\sqrt{\varepsilon_{\mathrm{s}}}\right)^{2}+r_{2}^{2}}{N_{0}}\right] .
\end{aligned}
$$

The Pdf of the phase $\theta_{r}$ is obtained by a change in variables from $(\mathrm{r} 1, \mathrm{r} 2)$ to $\left(\mathrm{A}, \theta_{r}\right)$ where :

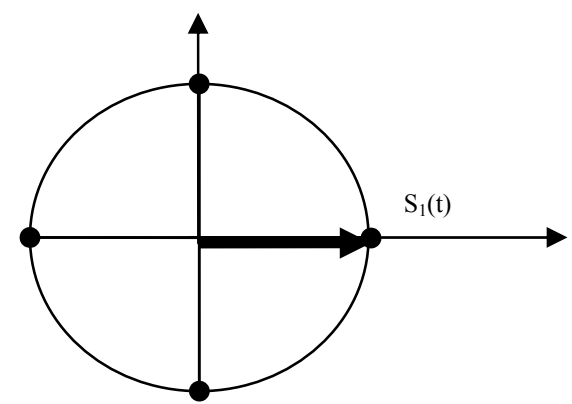

Fig.6 The HPSK vector constellation

$$
\mathrm{A}=\sqrt{r_{1}^{2}+r_{2}^{2}} \quad, \theta_{r}=\tan ^{-1} \frac{r_{2}}{r_{1}}
$$

Hence the joint $\operatorname{Pdf} P(A, \theta r)$ is

$$
P\left(A, \theta_{r}\right)=A\left(\frac{1}{\pi N_{o}}\right) \exp \left[-\frac{\left(A^{2}+\varepsilon_{s}-2 \sqrt{\varepsilon_{s}} A \cos \theta_{r}\right)}{N_{o}}\right] .
$$

Integration of $P(A, \theta r)$ over the range of $\mathrm{A}$ yields $\mathrm{P}(\theta \mathrm{r})$ that is

$$
\begin{aligned}
& P_{\theta_{r}}\left(\theta_{r}\right)=\int_{0}^{\infty} P(A, \theta r) d A \\
& =\frac{1}{2 \pi} e^{-2 \gamma_{s} \sin ^{2} \theta} \int_{0}^{\infty} A e^{-\frac{\left(A-\sqrt{4 \gamma_{s}} \cos \theta\right)^{2}}{2}}
\end{aligned}
$$

Where $\gamma_{s}=\frac{\varepsilon_{s}}{N_{0}}$ is the symbol SNR and $\varepsilon_{s}$ is the energy of the HPSK signal

For large values of $\gamma_{s}>1$ and $\left|\theta_{r}\right| \leq \frac{\pi}{2}, \mathrm{P} \theta_{r}\left(\theta_{r}\right)$ is well approximated as:

Where

$$
\begin{aligned}
& \mathrm{P} \theta_{r}\left(\theta_{r}\right)=\frac{1}{2 \pi} e^{-2 \gamma_{s} \sin ^{2} \theta_{r}} . I \\
& I=\int_{0}^{\infty} A \exp \left(-\frac{\left(A-\cos \theta r \sqrt{4 \gamma_{s}}\right)^{2}}{2} d A\right.
\end{aligned}
$$

$$
I=2 \cos \theta r \cdot \sqrt{\pi} \cdot \sqrt{2 \gamma s}
$$




$$
\begin{aligned}
& I=\cos \theta_{r} \cdot \sqrt{2 \pi} \cdot \sqrt{4 \gamma_{s}} \ldots \ldots \ldots \ldots \ldots \\
& P_{\theta_{r}}\left(\theta_{r}\right)=\sqrt{\frac{2 \gamma_{s}}{\pi}} \cos \theta_{r} \cdot e^{-2 \gamma_{s} \sin ^{2} \theta_{r}} \ldots \ldots . .
\end{aligned}
$$

When $\mathrm{s} 1(\mathrm{t})$ is transmitted a decision of error is made if the noise causes the phase to fall outside the range $[-\pi / 4 \pi / 4]$, and hence the probability of a symbol error is

$$
P_{4}=1-\int_{\frac{-\pi}{4}}^{\frac{\pi}{4}} P_{\theta r}(\theta r) d \theta r \ldots \ldots \ldots .
$$

By substituting for $P_{\theta_{r}}\left(\theta_{r}\right)$ in equation (31)

Performing the change of variables, we find:

$$
P_{4}=1-\int_{\frac{-\pi}{4}}^{\frac{\pi}{4}} \sqrt{\frac{2 \gamma_{s}}{\pi}} \cos \theta_{r} \cdot e^{-2 \gamma_{s} \sin ^{2} \theta_{r}} d \theta r
$$

Performing the change of variables from $\theta_{r}$ to $\mu$

$\mu=\sin \theta r \cdot \sqrt{2 \gamma_{s}}$ We find

$P 4=2 Q\left(\sqrt{2 \gamma_{s}} \sin \left(\frac{\pi}{4}\right)\right.$

$$
P 4=2 Q\left(\sqrt{2 \gamma_{s} \sin ^{2}\left(\frac{\pi}{4}\right.}\right)=2 Q\left(\sqrt{\gamma_{s}}\right)
$$

Since $\varepsilon_{s}$ in the case of HPSK is equal to : $\varepsilon_{s}=2\left(k \varepsilon_{b}\right)=4 \varepsilon_{b}$

$$
\therefore P 4=2 Q\left(\sqrt{\frac{4 \varepsilon_{b}}{N_{0}}}\right) \text {. }
$$

Since the transmitted signals represented by the vector points of the final constellation are equally likely to be transmitted and since the 8-points are distributed around a circle consisting of two independent QPSK constellations with complex scrambling then the average probability of the symbol error in the case of two channels at different amplitudes is:

$$
\text { Psymbol }=\frac{1}{2}[P 1(e)+P 2(e)]
$$

Where P1 (e) is the probability of symbol error of the first constellation corresponding to $\phi_{1}=\theta_{1}-45, \mathrm{P} 2$ (e) is the probability of symbol error of the second constellation corresponding to $\phi_{2}=45-\theta_{2}$.

Substituting (34) into (35), we get

$$
\mathrm{Ps}=2 \mathrm{Q}\left(\sqrt{\frac{4 \varepsilon_{b}}{N_{0}}}\right) .
$$

The bit error probability in this case is: 


$$
\begin{array}{r}
\mathrm{Pb}=\mathrm{Q}\left(\sqrt{\frac{4 \varepsilon_{b}}{N_{0}}}\right) . \\
\mathrm{Or}, \quad \mathrm{Pb}=\mathrm{Q}(\sqrt{4 \gamma}) \ldots \ldots .
\end{array}
$$

Where $\gamma$ is the SNR (=eb/No). Equation (38) shows an improvement of the BER of HPSK over that of QPSK.

\section{The performance of HPSK signal over a generalized Nakagami -m channel}

The mobile communication channel is noisy, multipath and is subjected to fading. The channel fading conditions depend on the propagation conditions and the clutter types the waves propagate through. In some cases the fading can be more severe than Rayleigh, while in other cases where line of sight or near line of sight conditions is available, the signal will be more stable. The Nakagami distribution is shown to fit results more generally than other distributions [4]. In this section we will evaluate the average BER of HPSK systems subjected to Nakagami fading. We will evaluate the expected value of the conditional $\mathrm{Pb}$ as given by equation (38) over Nakagami distribution.

In Nakagami fading channel the path amplitude probability density function is given by:

$$
f r(r)=\frac{2}{\Gamma(m)} \cdot\left(\frac{m}{\Omega}\right)^{m} \cdot r^{2 m-1} \exp \left(-\frac{m}{\Omega} r^{2}\right) .
$$

Where $\mathrm{m}$....is the fading parameter and it describes the fading severity it is defined as the ratio of moments or it is the ratio of the square of the mean signal power to the variance of the signal power

$$
m=\frac{\Omega^{2}}{E\left[r^{2}-\Omega\right]}
$$

And,

$$
\mathrm{m}>0.5
$$

$$
\Omega=E\left[r^{2}\right]
$$

The received signal power $\gamma$ follows gamma distribution and its pdf is given by

$$
f_{\gamma}(\gamma)=\left(\frac{m}{\Omega}\right)^{m} \cdot \frac{\gamma^{m-1}}{\Gamma(m)} \exp \left(-\frac{m}{\Omega} \gamma\right)
$$

Such that $\gamma \geq 0, m \geq 0.5$

The average probability of error will be given by

$$
P(e)=\int_{0}^{\infty} P(e / \gamma) f_{\gamma}(\gamma) d \gamma
$$

Or,

$$
P(e)=\int_{0}^{\infty} Q(\sqrt{4 \gamma}) f_{\gamma}(\gamma) d \gamma .
$$

The Integral of the average probability is evaluated in [5]. The average probability of error is finally given by: 
$P_{e}=\left(\frac{1}{2 \sqrt{\pi}}\right) \cdot \frac{\Gamma\left(m+\frac{1}{2}\right)}{\Gamma(m+1)}\left(\frac{2 m}{2 m+4 \Omega}\right)^{m} \cdot{ }_{2} F_{1}\left(\frac{1}{2}, m ; m+1 ; \frac{2 m}{2 m+4 \Omega}\right)$.

Where ${ }_{2} \mathrm{~F}_{1}(\mathrm{a}, \mathrm{b} ; \mathrm{c}, \mathrm{x})$ is the Gauss Hypergeometric Function [6] defined by:

$$
{ }_{2} F_{1}(a, b ; c, x)=\sum_{n=0}^{\infty} \frac{(a)_{n}(b)_{n}}{(c)_{n}} \frac{x^{n}}{\Gamma(n+1)}
$$

Or,

$$
{ }_{2} F_{1}(a, b ; c, x)=\frac{\Gamma(c)}{\Gamma(b) \Gamma(c-b)} \int_{0}^{1} t^{b-1}(1-t)^{c-b-1}(1-x t)^{-a} d t, \ldots \ldots \ldots c>b>0 .
$$

The last equation is the integral representation of $2 \mathrm{~F} 1$ and is valid under the assumption that $|x| \leq 1$. In our case, the integral representation reduces to:

$$
{ }_{2} F_{1}(1 / 2, m ; m+1 ; x)=\frac{\Gamma((m+1))}{\Gamma(m)} \int_{0}^{1} \frac{t^{m-1}}{\sqrt{(1-x t)}} d t
$$

The average BER can then be given by:

$$
P_{e}=\left(\frac{1}{2 \sqrt{\pi}}\right) \cdot \frac{\Gamma\left(m+\frac{1}{2}\right)}{\Gamma(m)} x^{m} \cdot \int_{0}^{1} \frac{t^{m-1}}{\sqrt{(1-x t)}} \cdot d t
$$

Where

$$
x=\frac{2 m}{2 m+4 \Omega} .
$$

The substitution $(t=\sin 2(\theta) / x)$, is useful to solve the integral. Finally, we have for arbitrary value of $\mathrm{m}$ :

$$
P_{e}=\left(\frac{1}{\sqrt{\pi}}\right) \cdot \frac{\Gamma\left(m+\frac{1}{2}\right)}{\Gamma(m)} \int_{0}^{\arcsin (\sqrt{x})} \sin ^{2 m-1} \theta . . d \theta .
$$

And for integer m, (51) will result into:

Where

$$
P_{e}=\left(\frac{1}{\sqrt{\pi}}\right) \cdot \frac{\Gamma\left(m+\frac{1}{2}\right)}{\Gamma(m)} \sum_{n=0}^{m-1}\left(_{n}^{m-1}\right) \frac{(-1)^{n}}{2 n+1}\left[1-y^{\left(n+\frac{1}{2}\right)}\right] \ldots
$$

$$
y=\frac{4 \Omega}{2 m+4 \Omega} .
$$

Special Cases:

The probability of error is computed for different value of $\mathrm{m}$. For the case of severe fading; $\mathrm{m}=1 / 2$ (the half Gaussian distribution). Equation (51) reduces to:

$$
P_{e}=\arcsin (\sqrt{x}) / \pi \text {. }
$$

And for $\mathrm{m}=3 / 2$, equation (51) will reduce to: 


$$
P_{e}=(\arcsin \sqrt{x}-\sqrt{x(1-x)}) / \pi .
$$

For $\mathrm{m}=1$ (the case of Rayleigh fading), the average probability of error in (52) reduces to:

$$
P_{e}=(1-y) / 2 \text {. }
$$

For $\mathrm{m}=2$, equation (52) will result to:

$$
P_{e}=\left(2-3 y+y^{3}\right) / 4
$$

And for $\mathrm{m}=3$ (very close to Rician fading), equation (52) will result to:

$$
P_{e}=\left(8-\left(15 y-10 y^{3}+3 y^{5}\right)\right) / 16 \text {. }
$$

\section{Results and Comments}

In this paper we come to an easy to evaluate expressions for the BER of HPSK performance in Nakagami fading channel. The cases of more severe fading than Rayleigh, Rayleigh and Racian fading are considered. Figure (7) shows the probability of error of HPSK for different values of $m$. The Cases of BPSK and QPSK are $6 \mathrm{~dB}$ and $3 \mathrm{~dB}$ worse than HPSK respectively.

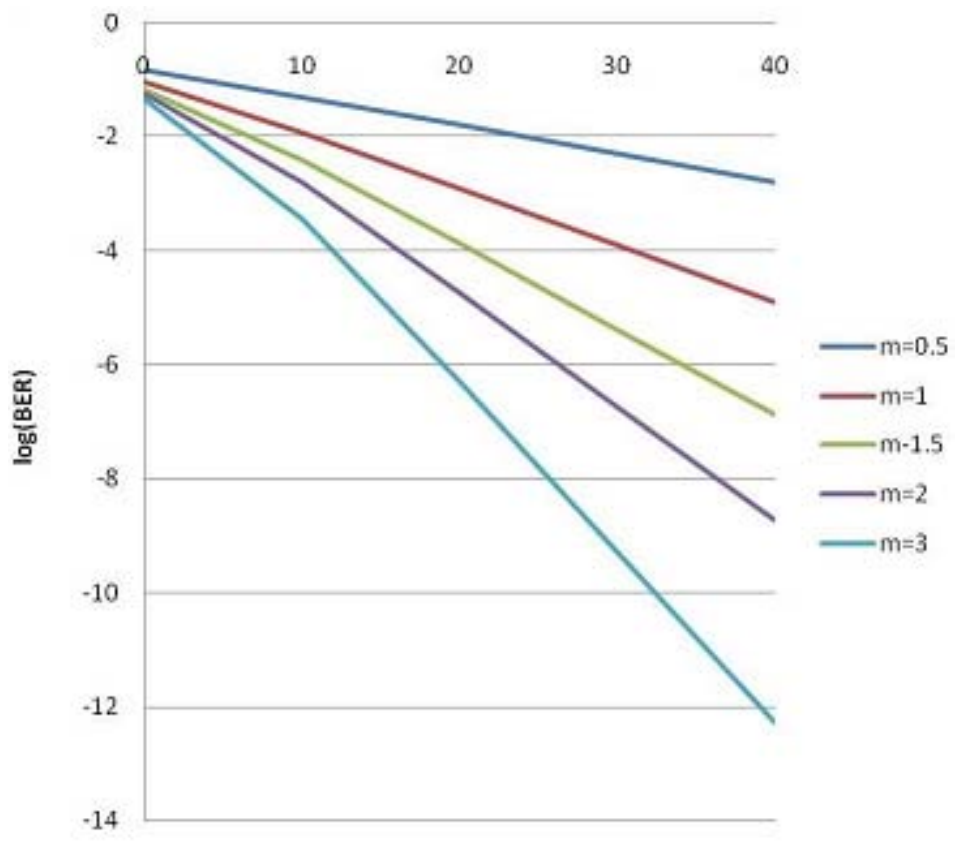

Fig.7 BER vs SNR(dB) of HPSK in Nakagami Fading Channels

\section{References}

[1] Agilent ,2003,"HPSK spreading for 3G", application note 2003.

[2] Proakis, 2001, J.G. Proakis, "Digital Communications " New York:McGraw-Hill, 2001.

[3] Lowel Hoover , 2005, "Deriving the quadratic modulators" PolyPhase microwave, AN101A \&AN102A, 2005 .

[4] U. Charach, "Reception Through Nakagami Fading Multipath Channels with Randam Delays", IEEE Transactions on Communications, vol. com-27, No. 4, April 1979.

[5] E.k. Al Hussaini and A. Al-Bassioni,"Performance of MRC Diversity Systems for the Detection of Signals with Nakagami Fading", IEEE Transactions on Communications, Vol. Com-33, No. 12, December 1985.

[6] Lary C. Andrews, Special Functions for Engineers and Applied and Applied Mathematician, Macmillan Publishing Company, 1985. 